\title{
Cuidados paliativos e a enfermagem ao paciente em unidade de terapia intensiva:
}

\section{Revisão integrativa}

\author{
Palliative care and nursing to the patient in an intensive care unit: An integrative review \\ Cuidados paliativos y enfermería al paciente en una unidad de cuidados intensivos: Una revisión
}

integradora

Recebido: 13/07/2021 | Revisado: 21/07/2021 | Aceito: 26/07/2021 | Publicado: 02/08/2021

Daniel Santos dos Santos
ORCID: https://orcid.org/0000-0002-6657-7924
Secretaria Municipal de Cacequi, Brasil
E-mail: danielsantosdoss@ hotmail.com
Silvana Carloto Andres
ORCID: https://orcid.org/0000-0001-6726-7947
Universidade Federal de Santa Maria, Brasil
E-mail: silvana.andres@ yahoo.com.br
Liane Bahú Machado
ORCID: https://orcid.org/0000-0002-1356-373X
Universidade Federal de Santa Maria, Brasil
E-mail: lianemachado61@gmail.com
Marjana Pivoto Reginaldo
ORCID: https://orcid.org/0000-0002-2260-8207
E-mail: marjanapivotto@gmail.com

\begin{abstract}
Resumo
A unidade de terapia intensiva (UTI), se define pelo conjunto de elementos funcionalmente agrupados, que se destina ao atendimento de pacientes graves e/ de riscos que precisam de assistência médica, enfermagem e fisioterapeutas continuamente, além de equipamentos tecnológicos modernos e recursos humanos especializados de diversas profissionais voltados à saúde. Para centralizar os cuidados aplicados ao paciente, o profissional de enfermagem que atua em terapia intensiva é desafiado pelas especificidades dessa assistência a cada paciente respeitando sua singularidade. Trata-se de um estudo descritivo com enfoque qualitativo do tipo revisão integrativa, a qual compreende a análise de pesquisas relevantes, permitindo sintetizar a informação sobre um determinado contexto e apontar lacunas que necessitam ser preenchidas a partir de um tema definido e característico. Embora os profissionais de enfermagem compreendem e reconheçam, a importância dos cuidados paliativos, existe um entendimento fragilizado, correlacionando-os apenas aos pacientes em sofrimento de vida, sendo assim os profissionais focam no enfoque no cuidado físico desse paciente e não nas outras necessidades do paciente como sujeito singular. Há ainda dificuldades para triar, prognosticar, reconhecer esse tipo de paciente, retardando o início dos cuidados paliativos, compartilhar o processo de tomada de decisão entre equipe multidisciplinar, planejar o cuidado de forma integral, fatores esses que influenciam do ponto de vista negativo para realização dos cuidados paliativos na UTI.
\end{abstract}

Palavras-chave: Enfermagem; Cuidados paliativos; Unidade de Terapia Intensiva.

\begin{abstract}
The intensive care unit (ICU) is defined by the set of functionally grouped elements, which is intended for the care of critically ill and/or at-risk patients who need medical care, nursing and physiotherapists continuously, in addition to modern technological equipment and specialized human resources of several professionals focused on health. In order to centralize the care applied to the patient, the nursing professional who works in intensive care is challenged by the specificities of this assistance to each patient, respecting their uniqueness. This is a descriptive study with a qualitative focus of the integrative review type, which comprises the analysis of relevant research, allowing the synthesis of information about a given context and pointing out gaps that need to be filled based on a defined and characteristic theme. Although nursing professionals understand and recognize the importance of palliative care, there is a weakened understanding, correlating them only to patients suffering from life, so professionals focus on focusing on the physical care of this patient and not on other patient needs as a singular subject. There are still difficulties in screening, prognosing, recognizing this type of patient, delaying the start of palliative care, sharing the decisionmaking process between a multidisciplinary team, planning care comprehensively, factors that negatively influence the achievement palliative care in the ICU.
\end{abstract}

Keywords: Nursing; Palliative care; Intensive Care Unit. 


\begin{abstract}
Resumen
La unidad de cuidados intensivos (UCI) se define por el conjunto de elementos agrupados funcionalmente, que está destinado a la atención de pacientes críticamente enfermos y / o en riesgo que necesitan atención médica, enfermería y fisioterapeutas de manera continua, además de modernos equipos tecnológicos y recursos humanos especializados de varios profesionales enfocados a la salud. Para centralizar los cuidados aplicados al paciente, el profesional de enfermería que trabaja en cuidados intensivos se ve desafiado por las especificidades de esta asistencia a cada paciente, respetando su singularidad. Se trata de un estudio descriptivo con enfoque cualitativo del tipo revisión integradora, que comprende el análisis de investigaciones relevantes, permitiendo la síntesis de información sobre un contexto dado y señalando los vacíos que deben ser llenados en base a un tema definido y característico. Si bien los profesionales de enfermería comprenden y reconocen la importancia de los cuidados paliativos, existe un entendimiento debilitado, correlacionándolos solo con pacientes que sufren de la vida, por lo que los profesionales se enfocan en enfocarse en el cuidado físico de este paciente y no en otras necesidades del paciente como un tema singular. Aún persisten dificultades en el cribado, el pronóstico, el reconocimiento de este tipo de pacientes, retrasar el inicio de los cuidados paliativos, compartir el proceso de toma de decisiones entre un equipo multidisciplinario, planificar la atención de manera integral, factores que influyen negativamente en el logro de los cuidados paliativos en la UCI.
\end{abstract}

Palabras clave: Enfermería; Cuidados paliativos; Unidad de Terapia Intensiva.

\title{
1. Introdução
}

O crescimento considerável da prevalência de patologias crônicas e incapacitantes e a demanda dos pacientes idosos, que geralmente são portadores de algumas síndromes demenciais de várias etiologias com graves implicações neurológicas e motoras, que procuram as instituições de saúde e são encaminhados para a unidade de terapia intensiva (UTI), tem ocasionado uma maior necessidade de serviços que prestem assistência de qualificação paliativa (Luiz et al., 2018).

A UTI, é definida por uma série de elementos funcionalmente agrupados, que está destinada a assistência para pacientes graves e dos grupos de riscos que precisam de atendimentos médicos, de enfermagem e fisioterapeutas de maneira contínua, além de equipamentos tecnológicos modernos e recursos humanos especializados de diversas profissionais voltados à saúde (Vargas \& Braga, 2017).

No Manual de Cuidados Paliativos, utilizando na maioria das UTI, a assistência prestada não baseia-se apenas em protocolos, mas seguem alguns princípios, que são fundamentados no atendimento humano e individual de cada paciente. O manual traz que não se usa o termo terminalidade, mas, sim doença que ameaça a vida (Academia Nacional de Cuidados Paliativos, 2020).

Posterior ao diagnóstico de patologias que colocam a vida em ameaça, iniciam-se a assistência voltada para os cuidados paliativos. A impossibilidade de cura não é destacada ou descartada, o foco é a existência ou não de tratamento que modifique a patologia, melhorando a qualidade dos cuidados prestados, tornando o mais humanizado possível, todo esse processo, fornecendo maior conforto viável (Santos et al., 2019).

É através deste protótipo que declara-se a divergência entre os cuidados críticos e cuidados paliativos, principalmente, ao se apresentar com os processos de morte nos cuidados ao fim de vida, que tornam necessárias decisões sobre os limites terapêuticos. A doença de câncer, por exemplo, embora possua características em estágios mais avançados, há maneiras terapêuticas para facilitar o seu prognóstico a partir do declínio progressivo da situação clínica do paciente durante sua última fase de vida, são corriqueiros os casos de aversão terapêutica clínica na UTI (Santos et al., 2017).

Nessa perspectiva, na contramão da tentativa de cura a todo custo, os cuidados paliativos surgem com o intuito de proporcionar cuidados ativos e totais ao paciente e sua família com intenção de ofertar dignidade e humanidade no tratamento, atendendo as suas necessidades durante o processo de morte (Rocha et al., 2017).

Para centralizar os cuidados aplicados ao paciente, o profissional de enfermagem intensitivista é desafiado pelas especificidades dessa assistência a cada ser humano respeitando sua singularidade. São pacientes fragilizados no aspecto das suas condições de saúde, expectativa de vida, demandante de diversos cuidados e sujeitos a uma série de intercorrências que 
exigem ações rápidas e eficazes das equipes multiprofissional e serviços especializados. Frequentemente, são também pacientes fragilizados psicologicamente pelas limitações, receios, distância da família e pelo próprio espaço característico desta unidade (Martins et al., 2019).

Quando pensamos na assistência prestada pelo profissional enfermeiro dentro do cenário da UTI, logo é evidente que a humanização, conforto, apoio, amparo, solidariedade, empatia e compaixão, são indispensáveis na realização da assistência paliativa. É preciso haver um bom acolhimento do paciente pelo enfermeiro, e este precisa dispor de habilidades para lidar com essa vivência, oportunizando um tratamento menos doloroso e mais digno (Santos et al., 2018).

Considera-se que o enfermeiro é o profissional que possui ligação diretamente ao paciente em todos os ambientes de cuidados, principalmente quando falamos em cuidados paliativos. Dessa forma, torna-se indispensável que esse profissional compreenda de maneira efetiva as necessidades e singularidades de cada paciente inserido nesse contexto. É necessário que haja muito engajamento e dedicação profissional para que seja possível uma verdadeira recuperação e promoção de saúde para todos os pacientes, inclusive para aqueles que não possuem perspectivas de cura e sobrevida.

Sendo assim, esta pesquisa teve como questão norteadora: Quais são as práticas de enfermagem oferecidas aos pacientes internados em UTI sob cuidados paliativos?

Portanto, objetivou-se analisar as publicações que abrangem o tema a acerca da assistência de enfermagem ao paciente em cuidados paliativos em UTI e relatar os cuidados para alcançar e planejar a assistência na perspectiva desses atendimentos indispensáveis.

\section{Metodologia}

Trata-se de um estudo descritivo com enfoque qualitativo do tipo revisão integrativa, a qual compreende a análise de pesquisas relevantes, permitindo sintetizar a informação sobre um determinado contexto e apontar lacunas que necessitam ser preenchidas a partir de um tema definido e característico (Mendes, 2008).

A revisão integrativa é uma pesquisa que provê elementos mais vastas e amplas de maneira sistemática, ordenada e abrangente, sobre uma questão, com finalidade de sintetizar resultados obtidos em pesquisa sobre temas ou questões (Ercole et al., 2015).

A pesquisa de revisão integrativa exige rigor cientifico, devendo o pesquisador percorrer todas as etapas, que asseguram o rigor do método exigido nesse tipo de pesquisa.

Sendo assim para a elaboração desta pesquisa revisão integrativa foi percorrido todas as etapas preconizadas e desenvolvidas seguindo seis fases distintas: 1) identificação do problema com definição da pesquisa; 2) estabelecimento de critérios de inclusão e/ou exclusão de estudos para a busca científica; 3) definição das informações a serem retiradas dos estudos; 4) avaliação dos estudos; 5) interpretação dos resultados e 6) apresentação da revisão/ síntese da informação. Para o estudo foi formulada a seguinte questão norteadora: Quais são as práticas de enfermagem oferecidas aos pacientes internados em UTI sob cuidados paliativos? A busca dos artigos foi realizada nas bases de dados Scielo, Literatura Latino-Americana e do Caribe em Ciências da Saúde (LILACS) e Base de Dados Brasileira de Enfermagem (BDENF).

Os descritores utilizados nas bases de dados foram: Cuidados Paliativos, Enfermagem e UTI, utilizando o operador boleano AND para ocorrência simultânea de assuntos. Os operadores booleanos são expressões usadas para fazer associações de vocábulos durante a pesquisa, os mais utilizados são: AND/ OR/ AND NOT. Podem-se combinar duas ou mais palavras/assuntos na busca, podendo também melhorar a pesquisa modificando os operadores ou acrescentando descritores (Bireme, 2009). Os descritores foram ajustados de diferentes modos com a finalidade de expandir a busca pelos artigos. Consideraram-se as alterações terminológicas e sinônimos. 
A seleção dos estudos foi realizada entre os meses de março a abril de 2021. Os critérios de inclusão para a seleção dos artigos científicos utilizados foram: a obtenção na íntegra, com resumo e texto completo na base de dados, de forma gratuita, artigos escritos em português, no período de 2017 a 2021, bem como manuais, cartilhas e protocolos disponíveis nas plataformas digitais de órgãos de saúde que abordem o tema. Foram excluídas publicações que não tratem do tema e artigos que se repetiram em duas bases.

Cabe ressaltar que durante o processo de aplicação dos critérios para incluir ou excluir estudos, foi realizado uma leitura dos títulos e dos resumos dos artigos, com o objetivo de excluir os que não se tratavam da temática pesquisada.

A pesquisa se isenta de ser aprovado em Comitê de ética, pois trata de dados já publicados, a revisão integrativa de literatura constitui na fidedignidade ás ideias dos autores, e a autenticidade das publicações.

\section{Resultados e Discussão}

Após a seleção dos estudos e leitura aplicados os critérios de inclusão previamente estabelecidos e assim distribuídos nas bases de dados selecionadas. Foram encontrados 58 estudos, dos quais 18 apresentaram-se repetidos em mais de um local, 12 estudos não estavam disponíveis na integra de forma gratuita, 15 estudos não contemplam o tema da pesquisa. Assim, das publicações elencadas, 13 abordavam o tema proposto e foram selecionadas para compor este estudo, e ficaram distribuídos da seguinte forma: LILACS (3), BDENF (3) e SciELO (4) elencando duas categorias para abranger no desenvolvimento: 1) Cuidados Paliativos na UTI e a legislação; 2) Cuidados Paliativos de enfermagem em UTI.

\section{Cuidados Paliativos na UTI e a legislação}

A UTI é o local onde se aplica profissionais especializados e os mais modernos recursos tecnológicos que têm como finalidade auxiliar no atendimento de pacientes com patologias graves ou incuráveis (Amaral et al., 2020).

A passagem entre os cuidados curativos e paliativos na UTI é um acontecimento habitual, o que não indica que a terapia intensiva tenha sido inadequada. Porém, quando isso acontece, é necessário reconhecer as fronteiras terapêuticas/medicamentosas e gerenciar as circunstâncias de forma baseada nos princípios que regem o cuidado humano, da bioética e da paliação. Porém, pode ser prevalente em profissionais e familiares, e até mesmo no próprio paciente, há esperança por bons resultados e melhora nas condições de saúde, principalmente nas primeiras horas de hospitalização por isso existe uma dificuldade e resistência em aceitar a situação e fazer essa transição da assistência necessária.

Os dilemas e conflitos éticos dos atendimentos em UTI surgem, principalmente, da falta de concordância com os preceitos dos cuidados paliativos e o cuidado do fim da vida, o que vai de encontro às recomendações da Organização Mundial da Saúde (OMS), ao tratar-se de uma patologia crônica que ameaça a continuidade da vida e a necessária implementação de ações paliativista ao viés de cuidados curativistas desde o seu diagnóstico, embora as vantagens destas sejam muito mais claras e evidentes quando a doença progride e alcança as condições elevadas de sofrimento (Martins et al., 2019).

No Brasil, as atividades relacionadas a Cuidados Paliativos ainda precisam ser regularizadas na forma de lei. Ainda imperam no Brasil um enorme desconhecimento e muito preconceito relacionado a esses cuidados, principalmente entre os médicos, profissionais de saúde, gestores hospitalares e poder judiciário. Ainda se confunde atendimento paliativo com eutanásia e há um enorme preconceito com relação ao uso de opióides, como a morfina, para o alívio da dor (Academia Nacional de Cuidados Paliativos, 2020).

Há uma evidente necessidade de associação precoce entre cuidados curativos e paliativos na UTI e esta tem sido abordada na literatura. E dentre as diversas estratégias para promover esse método, o investimento na educação permanente e continuada dos profissionais de saúde é unânime e essencial, pois dessa maneira, independente do modelo a ser utilizado, terão 
condições e qualificação de reconhecer o paciente de cuidados paliativos, melhor prognosticar e, mesmo, triar aqueles elegíveis ou não à terapia intensiva, proporcionando atendimento adequado (Bezerra et al., 2020).

O planejamento das ações preventivas e terapêuticas clínicas devem envolver tríade família-paciente-equipe, e neste ambiente de atendimentos, é importante que seja oferecido todo o apoio necessário como flexibilizar o horário de visitas, sempre que possível, controlar a dor e promover conforto ao paciente ou seja visar continuamente o bem-estar do paciente, dispensando tratamentos fúteis que adiem o morrer.

Almeida et al (2020) asseguram que é necessário fortalecer as estratégias que potencializam o conhecimento e a segurança dos profissionais que atuam no cenário da terapia paliativa. Neste mesmo estudo, foi comprovado que $80 \%$ dos profissionais estabelecem vínculos com os pacientes, todavia, reforça a necessidade de intensificar a qualidade da assistência através da educação continuada associada à prática assistencial.

Andres et al. (2021) evidenciaram a relevância e a precisão da inclusão dessa temática no decorrer da formação acadêmica dos profissionais de saúde, facilitando a aproximação e o conhecimento do tema, com objetivo do profissional lidar da maneira mais adequada com a morte e o morrer.

No clima hospitalar, a equipe de enfermagem convive por mais tempo com os pacientes em todos os ciclos da vida, incluindo o fim da vida, já que estes são condicionadas dos seus cuidados, desse modo o enfermeiro, por meio da Sistematização da Assistência de Enfermagem, devendo implementar e praticar estratégias de cuidado, com intuito de minimizar o impacto do tratamento, reduzir as dores físicas e mentais e proporcionar conforto (Freitas \& Carreiro, 2018).

As condutas paliativistas inovadoras ainda não possuem grande aceitação e adesão por parte da maioria dos profissionais, os quais são focados na assistência tradicional apenas curativista. Daí a relevância em fortalecer as pesquisas à respeito deste tema, com objetivo de provocar nestes, a vontade de conhecer e compreender os parâmetros de elegibilidade para paliação, preservar a implementação dos cuidados paliativos e o interesse prioritário de minimizar sofrimento em detrimento da distanásia (Meireles et al., 2020).

O cuidado multidisciplinar baseado em medidas farmacológicas e não farmacológicas, é indispensável e necessário, neste cenário, se faz importante o conhecimento profundo das medidas farmacológicas que proporcionam o alívio da dor e dos sintomas e que a equipe atue de forma coordenada, e na aplicação adequada das medidas não farmacológicas como o implemento das Práticas Integrativas e Complementares em saúde.

É fundamental a participação e engajamento de cada integrante da equipe, para que haja a prestação dos cuidados paliativos com excelência e efetividade. A "enfermagem paliativa" requer do enfermeiro uma assistência humanizada, sistematizada e integralizada, por meio do planejamento e estabelecimento de estratégias que tenham como resultado a maior autonomia para o paciente sobre sua própria vida e patologia; e também precisa que haja a habilidade para vincular-se com o paciente e seus familiares, e estes precisam ser ponderados como um constituinte primordial de cuidado para alcançar maior acolhimento e humanização na assistência (Lima et al., 2017).

É inevitável, em nossas vidas, a constante evidência da morte e somos cientes de que é um acontecimento natural do ciclo de vida de todos os seres humanos, porém, raramente é aceitável, em especial quando abrange os sentimentos de todo o contexto familiar. Sendo assim, o trabalho da equipe multidisciplinar de saúde é um agente facilitador da abordagem por meio de informações e orientações mais apropriadas à família e ao paciente, proporcionando dessa maneira uma capacidade superior de ofertar conforto e alívio das angústias vivencias (Andres, et al., 2021). 


\section{Cuidados Paliativos de enfermagem em UTI}

No universo das doenças incuráveis, crônicas ou "terminais" os profissionais de saúde, são diariamente instigados a refletir sobre o real significado/sentido da vida e da morte; bem como em até que ponto se pode avançar nos procedimentos e intervenções que dão o suporte vital (Façanha \& Maluf, 2017).

Portanto, pode-se afirmar que a enfermagem está intensamente ligada aos princípios filosóficos dos cuidados paliativos, apesar de não ser a única profissão que cuida, inegavelmente, é a profissão que tem mais oportunidade de cuidar, incorporando essa função como objetivo essencial e fundamental da sua prática (Santos et al., 2018).

Silva et al. (2018) reforçam que ao contrário da visão curativista, na paliativa o foco principal é o sujeito. O paciente passa a ser visto de maneira integral, um ser que possui capacidade de compreender sua probabilidade terapêutica, apoderar-se de suas vontades e decisões, e deve ser respeitados seus princípios. Comprovou-se que os cuidados de enfermagem para os pacientes em cuidados paliativos, estão concentrados no paciente e também nos familiares, com o intuito de minimizar e controlar o sofrimento físico, psicossocial e espiritual, tendo como foco realizar um cuidado integral e ideal com foco no contexto de modo geral (Silva et al., 2020).

Atualmente, a atenção aos sintomas e a relevância do tratamento e dos cuidados de enfermagem, precisam ser ponderados como habilidades e domínios particulares para a recuperação do paciente e família, para terapêutica e cuidados efetivos (Kanno et al., 2018).

Nesse sentido, integrar as formas dos cuidados paliativos como uma filosofia de cuidado também no cenário da UTI, fundamentada por ser um direito do paciente e dever da equipe de saúde oferecê-los, sendo indispensável um atendimento qualificado pelos profissionais que estão atuando nesse ambiente, de preferência a equipe multiprofissional, com o intuito de ofertar uma assistência com olhar holístico e individual, que respeite dignidade e garanta o bem-estar de todos os seres humanos (Luiz et al., 2018).

O princípio dos cuidados paliativos é ser especial e individual, uma vez que o enfermeiro precisa compreender quando o paciente inicia a fase terminal da vida para, os cuidados paliativos incidem em fornecer um cuidado integralizado para pacientes cuja patologia é gradativa e incurável, garantir o controle da dor e dos outros sintomas, de maneira farmacológica ou não farmacológica, bem como a redução das dificuldades psicológicas, sociais e espirituais (Santos et al., 2017).

A terminalidade pode ser considerada, como a fase mais difícil para o ser humano onde, em meio a muitas tecnologias, tratamentos, tentativas, a certeza da morte se torna cada dia mais real em seu dia a dia. Nesse sentido, exige um acompanhamento profissional bastante responsável, treinado e eficaz, fazendo com que os conhecimentos técnicos sejam ligados às práticas humanas objetivando proporcionar uma assistência qualificada aos pacientes em atendimentos, a enfermagem deve estar preparada para enfrentar e aprender que além das tecnologias existentes, há também a assistência de saúde humanizada (Rodrigues et al., 2017).

Portanto, é essencial que nesse processo, como exigência ética e moral, o respeito pela autonomia do ser humano e seu direito de decidir sobre o tratamento, além da justiça e a altruísmo que embasam a subsidiam a ética da enfermagem e que são essenciais para alcançar o bem-estar integral da pessoa (Freitas \& Carreiro, 2018).

Então, comunicar-se os familiares do paciente, esclarecer questionamentos, constituir um vínculo terapêutico, para garantir o prosseguimento da terapêutica. O enfermeiro, enquanto profissional em contato amplo com o paciente em cuidados paliativos, deve ainda gerar/ proporcionar a qualidade de vida nessa etapa de adoecimento (Bezerra et al., 2020). 


\section{Conclusão}

Apesar dos profissionais da saúde compreendem e reconheçam, a relevância da assistência prestada de forma qualificada e responsável em todos os contextos, especialmente neste em questão, há uma fragilidade importante no entendimento do mesmo, relacionando-os apenas aos seres humanos em sofrimento de vida, sendo assim os profissionais focam somente no cuidado físico desse paciente e não nas outras necessidades do ser humano como sujeito individual e singular.

Existem ainda dificuldades para acolher, triar, reconhecer e prognosticar, esses tipos de pacientes, retardando o início da assistência paliativa. Compartilhar o processo de tomada de decisão entre equipe multidisciplinar, realizar o planejamento do cuidado de maneira integralizada são fatores que influenciam do ponto de vista negativo para realização dos cuidados paliativos na UTI.

Destacam-se ainda, o déficit na formação acadêmica de profissionais sobre essa temática, ausências e falhas na comunicação entre as equipes multiprofissionais e especializadas, bem como os familiares. Sugere-se ainda, que sejam publicados cada vez mais estudos abrangendo essa temática tão relevante na vida dos profissionais, pacientes e familiares, pois, quanto mais a atuação profissional for embasada cientificamente, mais qualidade terá a assistência prestada, tanto para os pacientes, quanto para os familiares dos mesmos.

\section{Referências}

Almeida, P. F., et al. (2020). A relação entre o enfermeiro e o paciente nos cuidados paliativos oncológicos. Braz. J. Hea. Rev; (3) 2, 1465-1483.

Amaral, L. V. A., et al. (2020) Cuidados paliativos com a família de pacientes neonatos: um estado da arte. In: $13^{\circ}$ Congresso Nacional de Psicologia da Saúde-Actas. 323-31.

Andres, S. C. et al. (2021) Assistência de enfermagem aos pacientes em cuidados paliativos. Rev Research, Society and Development; (10) 6.

Academia Nacional de Cuidados Paliativos e Cuidados Paliativos no Brasil. (ACNP) (2020).

Bezerra, A. C et al. (2020) Palliative care in nursing in the intensive care unit: integrative review. Portuguese Rev Enferm UFPI; (9).

Bireme (Brasil) (2009). Biblioteca Virtual em Saúde - Tutorial de Pesquisa Bibliográfica. 24 p.

Ercole, F. F et al. (2015) Integrative review versus systematic review. Revista Mineira de Enfermagem; 18 (1) 9-12.

Façanha, T. R. S \& Maluf, F. (2017) A presença do ensino da bioética na enfermagem. Revista Pró-UniverSUS; (1) 8, 17-25.

Freitas, G. C. C., \& Carreiro, M. A. (2018) Cuidados paliativos na Unidade de Terapia Intensiva: a ética na assistência do enfermeiro intensivista. Revista PróUniverSUS; 09 (1): 86-92.

Kanno, P. H et al. (2018) Development and Validity of the Nursing Care Scale and Nurse`s Difficulty Scale in Caring fo Dying Patients With Cancer and Their Families in General Hospitals in Japan. Feature Article; 20 (6): 1-9.

Lima, S. F., et. al. (2017). Representações sociais sobre o cuidado paliativo entre profissionais de enfermagem. Rev. enferm. UFPE on line, 11(supl.5): 19801988, ISSN: 1981-8963.

Luiz, M. M., et al. (2018) Cuidados paliativos em enfermagem ao idoso em UTI: uma revisão integrativa. Revista Fundamental Care Online; 10 (2).

Martins, F. R., et al (2019) Necessidades de qualificação do processo de trabalho da Enfermagem em UTI Pediátrica. Id on Line Rev.Mult. Psic; (13) 43.

Meireles, D. S., et al. (2020) Assistência de enfermagem ao idoso em cuidados paliativos: um relato de experiência. Braz. J. of Develop; (6) 6, $40854-40867$.

Mendes, K. D. S., et al. (2008) Revisão integrativa: método de pesquisa para a incorporação de evidências na saúde e na enfermagem. Texto contexto enfermagem.

Rocha, R. C. N. P. et al. (2017) Experiências e necessidades espirituais do familiar cuidador de paciente em atenção paliativa oncológica.

Rodrigues, G. G. F. et al. (2017). Cuidados paliativos direcionados ao cliente oncológico: estudo bibliométrico. Rev. enferm. UFPE (on-line), 1349-1356.

Santos, C. E et al. (2018) Análise da Escala de Perroca em unidade de cuidados paliativos. Revista da Escola de Enfermagem da USP; (52).

Santos, D. C. L., et al. (2017) Planejamento da assistência ao paciente em cuidados paliativos na terapia intensiva oncológica. Acta paul. Enferm; (30) 3.

Santos, A. L. N., et al. (2018) Cuidados Paliativos Prestados Pelo Enfermeiro ao Paciente Oncológico. Dê Ciência em Foco. 2(1): 63-77. 
Research, Society and Development, v. 10, n. 9, e53810918209, 2021

(CC BY 4.0) | ISSN 2525-3409 | DOI: http://dx.doi.org/10.33448/rsd-v10i9.18209

Santos, R. J. L. L., et al. (2019) O enfermeiro e os cuidados paliativos proporcionados ao idoso terminal internado em UTI. Brazilian Journal of health Review; (2) 6.

Silva, F. C. F., et al. (2020). Assistência de enfermagem a pacientes com câncer em cuidados paliativos: revisão integrativa. Revista Enfermagem Atual In Derme. 90-21.

Silva, H. A., et al. (2018). Intervenção em cuidados paliativos: conhecimento e percepção dos enfermeiros. Rev enferm UFPE on line, 12(5):1325-30, ISSN: 1981-8963.

Vargas, D. \& Braga, A. L. (2017) O Enfermeiro de Unidade de Tratamento Intensivo: Refletindo sobre seu Papel. 